\section{Image Processing Software}
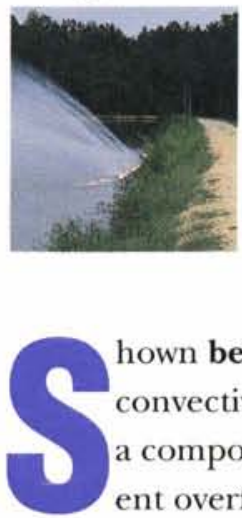
hown below is a satellite image of a convective cloud system over Alaska, a composite made from four different overflights by a polar orbiting satellite. The image typifies the type of satellite data collected by the National Weather Service (NWS) Alaska Region, Anchorage. To convert the streams of raw data into

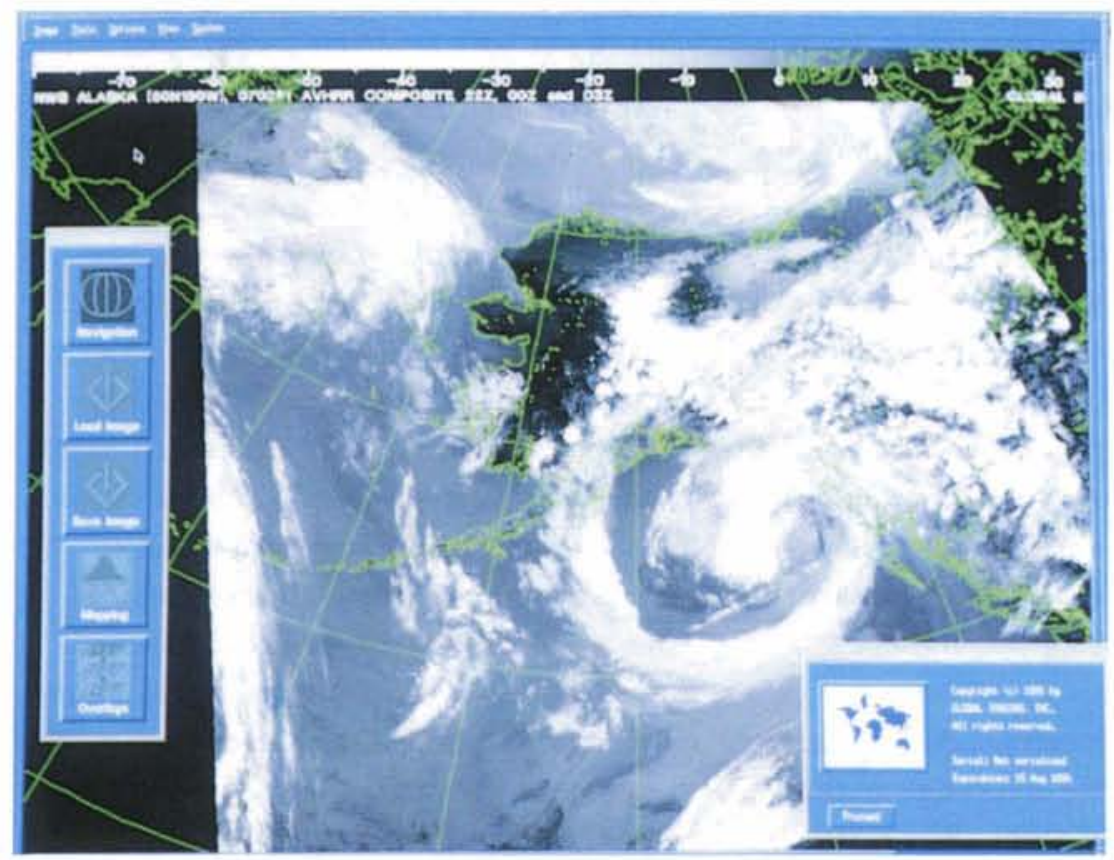

environmental products, NWS uses a spinoff Global System 9000 image processing system marketed by Global Imaging, Inc., Solana Beach, California.

Global System 9000 combines HP Apollo workstation hardware, manufactured by Hewlett-Packard Company, Palo Alto, California, and advanced Global Imaging software to offer, according to Global Imaging literature, "Cost-effective imaging software in a workstation environment incorporating capabilities previously found only in supercomputer or mainframe systems." Designed exclusively for the HP 9000 family of computers, Global System 9000 is intend- ed for a wide variety of applications in the fields of remote sensing, resource exploration, medicine, education, oceanography and military reconnaissance.

The spinoff element in Global System 9000 is the Global Applications Executive $^{\mathrm{TM}}$ (GAE) software package, an enhanced version of the NASA developed Transportable Applications Executive (TAE). Originally developed by Goddard Space Flight Center to support remote sensing and image processing applications, TAE was designed to lower the cost of system development by providing software and structures for commonly recurring requirements, such as menu and command interfaces, information displays, parameter processing, error reporting and on-line help. Over the years, TAE has evolved from a traditional command and menu-oriented system to a state-of-the-art user interface development system supporting highresolution-graphic workstations.

Global Imaging's version of the NASA software - GAE - "makes the system easy to learn and even easier to use." GAE can be operated in one of three interchangeable modes; menu, command or tutor. In the menu mode, the user selects from a list of application functions. In command, the user communicates with the system in simple English commands. In the tutor mode, the user is prompted for all parameters that must be supplied to an application program.

Among Global Imaging's customers, in addition to NWS, are the National Bureau of Standards, the Scripps Institute of Oceanography, universities, hospitals and industrial corporations.

TMGlobal Applications Executive is a trademark of Global Imaging, Inc. 